\title{
Automated Vehicles as a Space for Work \& Wellbeing
}

\author{
Bastian Pfleging \\ b.pfleging@tue.nl \\ Eindhoven University of Technology \\ Eindhoven, Netherlands \\ LMU Munich \\ Munich, Germany
}

\author{
Andrew L. Kun \\ andrew.kun@unh.edu \\ University of New Hampshire \\ Durham, NH, USA
}

\author{
Orit Shaer \\ oshaer@wellesley.edu \\ Wellesley College \\ Wellesley, MA, USA
}

\begin{abstract}
The objective of this $\mathrm{CHI}$ course is to provide $\mathrm{CHI}$ attendees with an introduction and overview of the rapidly evolving field of automotive user interfaces (AutomotiveUI). The course will focus on UI aspects in the transition towards automated driving. In particular, we will also discuss the opportunities of cars as a new space for non-driving-related activities, such as work, relaxation, and play. For newcomers and experts of other HCI fields, we will present the special properties of this field of $\mathrm{HCI}$ and provide an overview of new opportunities, but also general design and evaluation aspects of novel automotive user interfaces.
\end{abstract}

\section{CCS CONCEPTS}

- Human-centered computing $\rightarrow$ Human computer interaction (HCI); Ubiquitous and mobile computing.

\section{KEYWORDS}

Automotive user interfaces; non-driving-related activities; manual and automated driving; work and wellbeing.

\section{ACM Reference Format:}

Bastian Pfleging, Andrew L. Kun, and Orit Shaer. 2021. Automated Vehicles as a Space for Work \& Wellbeing. In CHI Conference on Human Factors in Computing Systems Extended Abstracts (CHI '21 Extended Abstracts), May 8-13, 2021, Yokohama, Japan. ACM, New York, NY, USA, 3 pages. https: //doi.org/10.1145/3411763.3445012

\section{INTRODUCTION}

In recent years, driving a car became more than the activity of maneuvering the car to a destination. In addition to the primary driving task, drivers also want to perform non-driving-related tasks [14, 19] including the operation of safety and comfort functions. Also, drivers want to be entertained (e.g., listen to music) or communicate while on the go. With the release of vehicles in different levels of automation, we expect this demand to become even more relevant and pressing. This opens the opportunity for novel activities in the car, such as work, play, and relaxation.

Already today, drivers use mobile devices in the car for these tasks. While driving manually, this can distract them from their primary driving task, posing humans at risk. Thus, the challenge for

Permission to make digital or hard copies of part or all of this work for personal or classroom use is granted without fee provided that copies are not made or distributed for profit or commercial advantage and that copies bear this notice and the full citation on the first page. Copyrights for third-party components of this work must be honored. For all other uses, contact the owner/author(s).

CHI '21 Extended Abstracts, May 8-13, 2021, Yokohama, Japan

(c) 2021 Copyright held by the owner/author(s).

ACM ISBN 978-1-4503-8095-9/21/05.

https://doi.org/10.1145/3411763.3445012 researchers and developers is to enable such non-driving-related tasks in the car in the transition to automated cars, while ensuring driving safety and comfort.

In contrast to our prior courses at CHI, MobileHCI, and MUM [15, $16,18]$, we will now especially focus on the transition to automated driving $[6,7]$ and how this makes the car an interesting and novel area for $\mathrm{HCI}$ research and applications, e.g., related to work, play, and relaxation. We provide an introduction to automotive UIs and highlights the special properties of this HCI sub-field. By outlining the requirements, the design, and evaluation of automotive UIs, we discuss the specific requirements for in-car applications.

\section{BENEFITS}

The objective of this course is to provide newcomers to the field of AutomotiveUI with an introduction and overview of the field and show how it is different from others. An intensive discussion of the requirements, trends, and challenges will help participants to get an overview and might be the basis for new research ideas. Especially with automated driving, we see the car as a platform that becomes more and more important for interactive applications and, thus, an important focus for human-computer interaction practice and research. We want attendees to leave with a fresh perspective on the field and inspire them to build the next generation of in-vehicle interfaces or services.

\section{INTENDED AUDIENCE}

We target a broad audience including AutomotiveUI novices (students, industrial / academic researchers), but also researchers, practitioners, \& designers with experiences in creating AutomotiveUIs. With driving automation, we see the car as a novel platform for interactive systems, which is interesting for attendees of a variety of backgrounds.

\section{PREREQUISITES}

The expected audience should have a basic knowledge of HCI. This could be a previously attended course or a basic university lecture or experiences from prior projects in this domain. There are no additional prerequisites.

\section{CONTENT \& PRACTICAL WORK}

During the course we first provide an overall introduction to $\mathrm{Au}-$ tomotiveUI. This includes a discussion of important terms, such as driving task, driver distraction, vehicle systems, and automation levels. In this part, we also discuss the influence of in-vehicle activities on driving. 
As part two, we provide an overview of best practices for the design of in-car systems, including principles, guidelines, and standards. We take this as a basis to also give deeper insights into the design process of such interfaces, enriched with practical experiences from previous projects.

In the third part of the course, the participants learn how applications and interfaces can be evaluated during design. This includes the explanation of various evaluation measures, different testing procedures (e.g., Lane Change Task, Detection Response Task, and field trials), and a variety of parameters related to designing studies.

For the fourth part, we elaborate the requirements and expected changes and opportunities for future automated driving situations. Also, based on Dagstuhl Seminars \#16262 and \#19132, this includes a discussion and hands-on session on the possibilities that arise when driving becomes highly or fully automated such as work, play, and relaxation.

As a concluding part, we outline current challenges and expected trends [9] with regard to AutomotiveUI and the more general topic of human mobility.

\section{PRESENTATION FORMAT \& COURSE ADVERTISEMENT}

We present this course as an interactive course with slides, videos, and group discussions. We successfully used this format for AutomotiveUI courses at CHI '16 and '17 [15, 16], MobileHCI '17 [18], MUM '18, and Mensch \& Computer '15. By applying active leaning methods, we strive to adapt the materials to the specific (existing) knowledge and interests of the audience.

The call for participation will be distributed via HCI, UX, and AutomotiveUI related mailing lists. We will further use our personal distribution lists.

\section{INSTRUCTOR BACKGROUNDS}

Bastian Pfleging (https://www.tue.nl/staff/b.pfleging) is assistant professor for Future Mobility at Eindhoven University of Technology, Netherlands. His research interests are user interfaces for future mobility, multimodal interaction, workload [17, 20], external interfaces for automated cars (e.g., $[1,3,5])$, and non-driving-related activities $[2,4,14,19]$. He was involved in co-organizing various workshops and courses (e.g., at AutomotiveUI, and CHI, [12, 13, 21]). Bastian is actively involved in organizing conferences like CHI, UIST, AutomotiveUI, MobileHCI and is steering committee member of AutomotiveUI.

Andrew L. Kun (http://www.andrewkun.com/) is professor of Electrical and Computer Engineering at the University of New Hampshire. His research focus is human-computer interaction in vehicles [8-11], as well as the use of visual behavior and pupil diameter measures [17] to assess and improve the design of interfaces. He is the steering committee chair of AutomotiveUI.

Orit Shaer (http://cs.wellesley.edu/ mobileoffice/) is Associate Professor of computer science and director of Media Arts and Sciences at Wellesley College. Her expertise is in designing, implementing and evaluating novel human-computer interactions including augmented reality (e.g. [23], and tangible interaction [22]. She served as program co-chair of the 2020 ACM Tangible, Embedded, and Embodied Interaction (TEI) conference.

\section{RESOURCES}

Additional details about the course as well as accompanying material will be published on our course website at https://ubisys. org/chi2021-course-future-cars/. It provides information about the course and links to related material, so that participants can get familiar with the scope of the subject and the goals of the course.

\section{ACKNOWLEDGMENTS}

Andrew Kun and Orit Shaer were supported in part by NSF grants CMMI-1840085 and CMMI-1840031.

\section{REFERENCES}

[1] Ashley Colley, Jonna Häkkilä, Bastian Pfleging, and Florian Alt. 2017. A Design Space for External Displays on Cars. In Proceedings of the 9th International Conference on Automotive User Interfaces and Interactive Vehicular Applications Adjunct (Oldenburg, Germany) (AutomotiveUI '17). Association for Computing Machinery, New York, NY, USA, 146-151. https://doi.org/10.1145/3131726.3131760

[2] Henrik Detjen, Bastian Pfleging, and Stefan Schneegass. 2020. A Wizard of Oz Field Study to Understand Non-Driving-Related Activities, Trust, and Acceptance of Automated Vehicles. In 12th International Conference on Automotive User Interfaces and Interactive Vehicular Applications (Virtual Event, DC, USA) (AutomotiveUI '20). Association for Computing Machinery, New York, NY, USA, 19-29. https://doi.org/10.1145/3409120.3410662

[3] Debargha Dey, Azra Habibovic, Bastian Pfleging, Marieke Martens, and Jacques Terken. 2020. Color and Animation Preferences for a Light Band EHMI in Interactions Between Automated Vehicles and Pedestrians. In Proceedings of the 2020 CHI Conference on Human Factors in Computing Systems (Honolulu, HI, USA) (CHI '20). Association for Computing Machinery, New York, NY, USA, 1-13. https://doi.org/10.1145/3313831.3376325

[4] Renate Häuslschmid, Bastian Pfleging, and Andreas Butz. 2017. The Influence of Non-driving-Related Activities on the Driver's Resources and Performance. In Automotive User Interfaces: Creating Interactive Experiences in the Car, Gerrit Meixner and Christian Müller (Eds.). Springer International Publishing, Cham, 215-247. https://doi.org/10.1007/978-3-319-49448-7_8

[5] Kai Holländer, Ashley Colley, Christian Mai, Jonna Häkkilä, Florian Alt, and Bastian Pfleging. 2019. Investigating the Influence of External Car Displays on Pedestrians' Crossing Behavior in Virtual Reality. In Proceedings of the 21st International Conference on Human-Computer Interaction with Mobile Devices and Services (Taipei, Taiwan) (MobileHCI '19). Association for Computing Machinery, New York, NY, USA, Article 27, 11 pages. https://doi.org/10.1145/3338286.3340138

[6] Christian P. Janssen, Linda Ng Boyle, Andrew L. Kun, Wendy Ju, and Lewis L. Chuang. 2019. A Hidden Markov Framework to Capture Human-Machine Interaction in Automated Vehicles. International fournal of Human-Computer Interaction 35, 11 (2019), 947-955. https://doi.org/10.1080/10447318.2018.1561789

[7] Christian P. Janssen, Shamsi T. Iqbal, Andrew L. Kun, and Stella F. Donker. 2019. Interrupted by my car? Implications of interruption and interleaving research for automated vehicles. International Journal of Human-Computer Studies 130 (2019), 221-233. https://doi.org/10.1016/j.ijhcs.2019.07.004

[8] Andrew L. Kun. 2018. Human-Machine Interaction for Vehicles: Review and Outlook. Foundations and Trends ${ }^{\circledR}$ in Human-Computer Interaction 11, 4 (2018), 201-293. https://doi.org/10.1561/1100000069

[9] Andrew L. Kun, S. Boll, and A. Schmidt. 2016. Shifting Gears: User Interfaces in the Age of Autonomous Driving. IEEE Pervasive Computing 15, 1 (Jan 2016), 32-38. https://doi.org/10.1109/MPRV.2016.14

[10] Andrew L. Kun, Alexander Shyrokov, and Peter A. Heeman. 2013. Interactions between human-human multi-threaded dialogues and driving. Personal and Ubiquitous Computing 17, 5 (2013), 825-834. https://doi.org/10.1007/s00779-0120518-1

[11] Andrew L. Kun, Jerry Wachtel, W. Thomas Miller, Patrick Son, and Martin Lavallière. 2015. User Interfaces for First Responder Vehicles: Views from Practitioners, Industry, and Academia. In Proceedings of the 7th International Conference on Automotive User Interfaces and Interactive Vehicular Applications (Nottingham, United Kingdom) (AutomotiveUI '15). ACM, New York, NY, USA, 163-170. https://doi.org/10.1145/2799250.2799289

[12] Alexander Meschtscherjakov, Manfred Tscheligi, Bastian Pfleging, Shadan Sadeghian Borojeni, Wendy Ju, Philippe Palanque, Andreas Riener, Bilge Mutlu, and Andrew L. Kun. 2018. Interacting with Autonomous Vehicles: Learning from Other Domains. In Extended Abstracts of the 2018 CHI Conference on Human Factors in Computing Systems (Montreal QC, Canada) (CHI EA '18). Association for Computing Machinery, New York, NY, USA, 1-8. https://doi.org/10.1145/ 3170427.3170614

[13] Alexander Meschtscherjakov, Manfred Tscheligi, Dalila Szostak, Sven Krome, Bastian Pfleging, Rabindra Ratan, Ioannis Politis, Sonia Baltodano, Dave Miller, 
and Wendy Ju. 2016. HCI and Autonomous Vehicles: Contextual Experience Informs Design. In Proceedings of the 2016 CHI Conference Extended Abstracts on Human Factors in Computing Systems (San Jose, California, USA) (CHI EA '16). Association for Computing Machinery, New York, NY, USA, 3542-3549. https://doi.org/10.1145/2851581.2856489

[14] Bastian Pfleging. 2017. Automotive User Interfaces for the Support of Non-DrivingRelated Activities. Ph.D. Dissertation. University of Stuttgart, Stuttgart, Germany. https://doi.org/10.18419/opus-9090

[15] Bastian Pfleging, Nora Broy, and Andrew L. Kun. 2016. An Introduction to Automotive User Interfaces. In Proceedings of the 2016 CHI Conference Extended Abstracts on Human Factors in Computing Systems (San Jose, California, USA) (CHI EA '16). ACM, New York, NY, USA, 961-964. https://doi.org/10.1145/2851581. 2856685

[16] Bastian Pfleging, Nora Broy, and Andrew L. Kun. 2017. An Introduction to Automotive User Interfaces. In Proceedings of the 2017 CHI Conference Extended Abstracts on Human Factors in Computing Systems (Denver, Colorado, USA) (CHI EA '17). ACM, New York, NY, USA, 1244-1248. https://doi.org/10.1145/3027063. 3027110

[17] Bastian Pfleging, Drea K. Fekety, Albrecht Schmidt, and Andrew L. Kun. 2016. A Model Relating Pupil Diameter to Mental Workload and Lighting Conditions. In Proceedings of the 2016 CHI Conference on Human Factors in Computing Systems (San Jose, California, USA) (CHI '16). ACM, New York, NY, USA, 5776-5788. https://doi.org/10.1145/2858036.2858117

[18] Bastian Pfleging, Andrew L. Kun, and Nora Broy. 2017. The Car as an Environment for Mobile Devices. In Proceedings of the 19th International Con ference on Human-Computer Interaction with Mobile Devices and Services (Vienna, Austria) (MobileHCI '17). ACM, New York, NY, USA, Article 63, 5 pages. https://doi.org/10.1145/3098279.3119916

[19] Bastian Pfleging, Maurice Rang, and Nora Broy. 2016. Investigating User Needs for Non-driving-related Activities During Automated Driving. In Proceedings of the 15th International Conference on Mobile and Ubiquitous Multimedia (Rovaniemi, Finland) (MUM '16). ACM, New York, NY, USA, 91-99. https://doi.org/10.1145/ 3012709.3012735

[20] Florian Roider, Sonja Rümelin, Bastian Pfleging, and Tom Gross. 2017. The Effects of Situational Demands on Gaze, Speech and Gesture Input in the Vehicle. In Proceedings of the 9th International Conference on Automotive User Interfaces and Interactive Vehicular Applications (Oldenburg, Germany) (AutomotiveUI '17). ACM, New York, NY, USA, 94-102. https://doi.org/10.1145/3122986.3122999

[21] Shadan Sadeghian Borojeni, Alexander Meschtscherjakov, Bastian Pfleging, Birsen Donmez, Andreas Riener, Christian P. Janssen, Andrew L. Kun, Wendy Ju, Christian Remy, and Philipp Wintersberger. 2020. Should I Stay or Should I Go? Automated Vehicles in the Age of Climate Change. In Extended Abstracts of the 2020 CHI Conference on Human Factors in Computing Systems (Honolulu, HI, USA) (CHI EA '20). Association for Computing Machinery, New York, NY, USA, 1-8. https://doi.org/10.1145/3334480.3375162

[22] Orit Shaer, Eva Hornecker, et al. 2010. Tangible User Interfaces: Past, Present, and Future Directions. Foundations and Trends ${ }^{\circledR}$ in Human-Computer Interaction 3, 1-2 (2010), 4-137. https://doi.org/10.1561/1100000026

[23] Hidde van der Meulen, Andrew L. Kun, and Orit Shaer. 2017. What Are We Missing?: Adding Eye-Tracking to the HoloLens to Improve Gaze Estimation Accuracy. In Proceedings of the 2017 ACM International Conference on Interactive Surfaces and Spaces (Brighton, United Kingdom) (ISS '17). ACM, New York, NY, USA, 396-400. https://doi.org/10.1145/3132272.3132278 